\title{
Evaluación de la percepción socio-emocional en personas con alexitimia
}

\section{Evaluation of socio-emotional perception in people with alexithymia}

\author{
Eduardo S. Martínez-Velázquez, Leopoldo G. Conde-Mota y Gregorio García-Aguilar \\ Benemérita Universidad Autónoma de Puebla, México
}

\begin{abstract}
Resumen
La alexitimia se define como la dificultad de identificar, analizar y expresar las experiencias emocionales. Se ha reportado que las personas con alexitimia presentan dificultades a nivel social y en la percepción de distintos estímulos emocionales, sin embargo los resultados no han sido consistentes.

Objetivos: identificar el efecto de palabras socio-emocionales (insultos, elogios y neutros) en la percepción de personas con alto y bajo nivel de alexitimia por medio de un Stroop emocional.

Método: Participaron 44 estudiantes de nivel licenciatura y se conformaron dos grupos con base en la escala TAS-20, un grupo con alto puntaje $\mathrm{GA}(>61)$ y otro con bajo puntaje de alexitimia $\mathrm{GB}(<28)$. Se evaluó la percepción de estímulos socio-emocionales por medio del Stroop emocional, presentados en bloques por categoría. Además, se registraron los puntajes clínicos de depresión, ansiedad y fobia social.

Resultados: Se observó que el GA tuvo mayores tiempos de reacción ante las palabras socio-emocionales en comparación con el GB. Se presentó también un mayor puntaje en las escalas clínicas en el GA que el GB.

Conclusiones: Los resultados sugieren un sesgo atencional en personas con alexitimia ante palabras socio-emocionales. Asimismo, la puntuación obtenida de las escalas se mostró a favor de la relación entre la alexitimia y los síntomas clínicos.
\end{abstract}

Palabras clave: Stroop emocional, Alexitimia, percepción emocional, estímulos socio-emocionales, regulación emocional

\begin{abstract}
Alexithymia has been defined as difficulties in identifying, analysing, and expressing emotional experiences. It has been reported that people with alexithymia have social difficulties and difficulties in perceiving different emotional stimuli. However, the results have not been consistent.

Objective: To identify the effect of socio-emotional words (insults, praise, and neutral words) on the perception of people with high and low levels of alexithymia using an emotional Stroop test.

Method: Participants were 44 undergraduate students. Two groups were formed based on the TAS-20 scale: one group with high GA scores (>61) and the other with low GB scores $(<28)$. The emotional Stroop test was used to assess the perception of socio-emotional stimuli presented in blocks by category. Scores on clinical depression, anxiety, and social phobia scores were recorded.

Results: Reaction times to socio-emotional words were longer in the GA group than those in the GB group. Scores on clinical measures were higher in the GA group than in the GB group.

Conclusions: The results suggest an attentional bias associated with a deficiency in emotional regulation(top-down) when people with alexithymia are presented with socio-emotional words. In addition, scores on the clinical measures suggest an association between alexithymia and clinical symptoms.
\end{abstract}

Keywords: Emotional Stroop test, alexithymia, emotional perception, socio-emotional stimuli, emotional regulation

Cómo citar este artículo: Martínez-Velázquez, E. S., Conde-Mota, L. G., \& García-Aguilar, G. (2020). Evaluación de la percepción socio-emocional en personas con alexitimia. Escritos de Psicología - Psychological Writings, 13(1), 13-22. https://doi.org/10.24310/espsiescpsi.v13i1.10074

Correspondencia: Eduardo Salvador Martínez-Velázquez. Facultad de Psicología. Benemérita Universidad Autónoma de Puebla (BUAP) Calle 3 Oriente No. 403. Puebla, Puebla. México. C.P. 72000. E-mail: eduardo.martinezvel@correo.buap.mx. E-mail del coautor Leopoldo Gerardo Conde Mota: psic.leopoldo.conde@gmail.com. E-mail del coautor Gregorio García-Aguilar: gregorio.garcia@correo.buap.mx 


\section{Introducción}

La alexitimia ha sido definida como la dificultad para identificar, analizar y expresar emociones (Bagby, Parker, y Taylor, 1994; Sifneos, 1973; van der Velde et al., 2014). Según diversos estudios clínicos, algunos autores han propuesto que la alexitimia está conformada por cuatro principales componentes interrelacionados: (1) dificultad para Identificar sentimientos y distinguirlos de sensaciones corporales; (2) dificultad para describir sentimientos; (3) una marcada escases de fantasía y; (4) un pensamiento orientado hacia lo externo (Bagby et al., 1994; Taylor, Bagby, y Parker, 1997). De este modo, se ha reportado que las personas con alexitimia pueden presentar dificultades a nivel emocional, interpersonal y social (Mallinckrodt y Wei, 2005; Rozenstein, Stein, y Latzer, 2018). Particularmente, se ha señalado que pueden presentar dificultades para reconocer y empatizar con los estados emocionales de otras personas (Taylor y Bagby, 2013). Asimismo, se ha encontrado que las personas con alexitimia suelen evitar las relaciones cercanas, y cuando se relacionan con otros, tienden a ser independientes o impersonales, de forma tal que la relación se vuelve superficial (Vanheule, Desmet, Rosseel, y Verhaeghe, 2007). Lo anterior se debe a una doble indiferencia interpersonal, al no tener expectativas de los demás y mostrar un nulo deseo por cumplir las expectativas de los otros (Vanheule et al., 2007). También, se ha reportado que presentan estilos interpersonales de apego, inseguros, fríos, y que manifiestan un comportamiento socialmente evasivo (Calderon, Samstag, Papouchis, y Saunders, 2019; Vanheule et al., 2007).

Se ha propuesto en la literatura que la etiología de la alexitimia puede ser multifactorial (Bermond, Oosterveld, y Vorst, 2015), y que involucra componentes neurológicos (Bermond, Vorst, y Moormann, 2006), ambientales (Freyberger, Künsebeck, Lempa, Wellmann, y Avenarius, 1985; Sifneos, 1988) o incluso genéticos (Picardi et al., 2011). Algunos autores han planteado que la alexitimia está relacionada con diversas patologías, entre las cuales se encuentran: la depresión (Grynberg, Luminet, Corneille, Grèzes, y Berthoz, 2010; Saarijärvi, Salminen, y Toikka, 2006), la ansiedad (Grynberg, Chang, Corneille, Maurage, Vermeulen, Berthoz, y Luminet, 2012; Hamaideh, 2017), el estrés (Hamaideh, 2017), entre otras. Sin embargo, otros autores consideran que la alexitimia es un rasgo estable de la personalidad que puede presentarse de manera independiente de patologías (Luminet, Rokbani, Ogez, y Jadoulle, 2007; Saarijärvi et al., 2006), por lo que los resultados aún no han sido concluyentes.

En relación con la evaluación de la alexitimia, se han propuesto distintas escalas que evalúan sus características (Bagby et al., 1994; Sifneos, 1973; Vorst y Bermond, 2001). Una de las más utilizadas ha sido la escala de Toronto; por sus siglas en inglés TAS-20 (Toronto alexitimic Scale) (Bagby et al., 1994) y está conformada por 20 ítems (Bagby et al., 1994).

La evaluación de la alexitimia por medio de las escalas ha permitido estudiar las dificultades de percepción emocional que las personas con dichos rasgos pudieran presentar (Donges, Kersting, y Suslow, 2014; Hobson et al., 2018). Por ejemplo, se ha reportado que las personas con alexitimia, evaluadas particularmente con el TAS-20, muestran una deficiencia en la percepción emocional de estímulos externos de tipo lingüístico y no lingüístico (Hobson et al., 2018). En este sentido, algunos estudios han reportado que ante las tareas no lingüísticas, las personas con alexitimia tienen dificultades para identificar expresiones faciales emocionales, que implican caras con expresiones de miedo, tristeza y enojo (Cook, Brewer, Shah, y Bird, 2013).

Con respecto a los estímulos emocionales lingüísticos, distintos autores han utilizado la prueba de Stroop emocional para evaluar la percepción emocional en personas con alexitimia (Lundh y Simonsson-Sarnecki, 2002; Mueller, Alpers y Reim, 2006; Pandey Rakesh, 1995; Parker, Taylor, y Bagby, 1993; Sánchez y Serrano, 1997), sin embargo, los resultados no han sido consistentes.

En un estudio se presentó a estudiantes universitarios un Stroop emocional en láminas que contenían palabras activadoras como: violación, pene, sida, víctima y vagina (Parker et al., 1993). Los autores encontraron que las personas clasificadas con alexitimia conforme al TAS-20, respondieron significativamente más lento que las personas sin alexitimia (Pandey Rakesh, 1995; Parker et al., 1993). Posteriormente, estos hallazgos fueron replicados en la India utilizando palabras Hindi (Pandey Rakesh, 1995).

Asimismo, en un estudio se presentó el Stroop emocional por computadora con palabras relacionadas con enfermedad, negativas y neutras en condiciones enmascaradas y no enmascaradas (Lundh y Simonsson-Sarnecki, 2002). Los autores reportaron que solo las personas con alexitimia tuvieron mayores tiempos de reacción ante las palabras de enfermedad en comparación con las negativas (Lundh y Simonsson-Sarnecki, 2002). Además, señalaron que el efecto pudo observarse solo al comparar los grupos extremos con base al puntaje del TAS-20 y en la condición no enmascarada (Lundh y Simonsson-Sarnecki, 2002). 
En conjunto, los resultados de los estudios descritos sugieren que la interferencia asociada con el Stroop emocional de las personas con alexitimia puede deberse a que presentan un mayor nivel de activación autónoma o sensibilidad inducida (Pandey Rakesh, 1995; Parker et al., 1993; Lundh y Simonsson-Sarnecki, 2002). Dicha activación puede ser estimulada por el contenido semántico de las palabras, ya sean activadoras (Pandey Rakesh, 1995; Parker et al., 1993) o relacionadas con enfermedad (Lundh y Simonsson-Sarnecki, 2002).

En contraste, otros estudios han observado resultados opuestos a los anteriores (Sánchez y Serrano, 1997; Mueller et al., 2006). En un estudio se presentaron por computadora palabras neutras (ej., armario, historia, idea) y activadoras (ej., accidente, bisturí, fracaso) a estudiantes universitarios con y sin alexitimia (Sánchez y Serrano, 1997). Los resultados indicaron que las personas sin alexitimia tuvieron mayores tiempos de reacción que las personas con alexitimia ante las palabras activadoras. Los autores interpretaron que las personas con alexitimia son menos hábiles que los no alexitímicos para reconocer estímulos emocionales (Sánchez y Serrano, 1997).

En la misma línea, Mueller et al., (2006) administraron un Stroop emocional por computadora en el que emplearon palabras negativas, positivas y relacionadas con síntomas corporales. La prueba fue aplicada a pacientes psicosomáticos de un hospital que fueron clasificados con alta y baja alexitimia conforme la prueba TAS-20 y la escala OAS (Haviland, Warren, y Riggs, 2000). Los autores encontraron un menor índice de interferencia en los pacientes con alta alexitimia que los de baja alexitimia ante palabras negativas y relacionadas con síntomas corporales (Mueller et al., 2006). Los resultados se basaron en la escala OAS y fueron interpretados como un déficit en el procesamiento del material emocional de las personas con alta alexitimia (Mueller et al., 2006).

En síntesis, los estudios de percepción emocional que comparan personas con mayor y menor nivel de alexitimia, han reportado resultados inconsistentes al emplear estímulos lingüísticos en el Stroop emocional. Algunos han mostrando un efecto de interferencia en las personas clasificadas con alexitimia (Pandey Rakesh, 1995; Parker et al., 1993; Lundh y Simonsson-Sarnecki, 2002), y otros en personas sin alexitimia (Sánchez y Serrano, 1997; Mueller et al., 2006).

Las inconsistencias de los estudios descritos podrían ser explicadas por las distintas metodologías utilizadas. Por ejemplo, el número y tipo de estímulos, la forma de aplicación de los estímulos, la edad o las características clínicas de los participantes. Un aspecto importante a resaltar es que los estímulos lingüísticos pueden ser menos activadores que el material visual de rostros, imágenes, o videos (Kissler, Assadollahi, y Herbert, 2006; Keil, 2006). Para lograr la activación de los estímulos lingüísticos, se requiere alcanzar un nivel de umbral que depende de varios factores como: el involucramiento en una tarea cognitiva, el estado del individuo, y la naturaleza e intensidad del estímulo emocional (Carretié et al., 2008). Estos factores podrían ser una posible explicación de los diferentes resultados reportados en los estudios de alexitimia revisados. Otra variable relevante, que particularmente fue distinta entre los estudios, fue la forma de presentar los estímulos. Los estudios que reportaron un efecto de interferencia en personas con alexitimia presentaron sus estímulos en bloques con distintas categorías, ya sean neutros y activadores (Pandey Rakesh, 1995; Parker et al., 1993)arousal words, and baseline stimuli. Both the alexithymic Ss and the non-alexithymic Ss took longer to color-name arousal words than to color-name neutral words and baseline stimuli. However, consistent with the view that alexithymia reflects a deficit in the ability cognitively to process and modulate emotional stimuli, the alexithymic Ss took longer than the non-alexithymic Ss to color-name arousal words. The groups did not differ significantly in their ability to color-name neutral words and baseline stimuli. (PsycINFO Database Record (c o relacionadas con enfermedades, negativas y neutras (Lundh y Simonsson-Sarnecki, 2002). Mientras que los estudios que encontraron un efecto de interferencia en personas sin alexitimia, presentaron sus estímulos en bloques mixtos que contenían distintas categorías; ya sean síntomas corporales, emociones negativas, positivas y neutras (Mueller et al., 2006); o activadores y neutras (Sánchez y Serrano, 1997).

En relación con la presentación de los estímulos, algunos estudios han reportado que la interferencia en el Stroop emocional es más consistente si los estímulos son presentados por bloques de una sola categoría, ya que los diseños con bloques mixtos pueden atenuar su efecto (McKenna y Sharma, 2004; Taake, Jaspers-Fayer, y Liotti, 2009). La interferencia por bloque es explicada por la influencia del estímulo amenazante previo, que es conocido como "efecto lento", mientras que la interferencia que se presenta en los bloques mezclados depende del estímulo inmediato, llamado "efecto rápido" (McKenna y Sharma, 2004). Así, parece ser que los estudios que encontraron un efecto de interferencia en las personas con alexitimia, fue por un efecto "lento" al presentar los estímulos por bloques de una categoría (Pandey Rakesh, 1995; Parker et al., 1993; Lundh y Simonsson-Sarnecki, 2002). Mientras que los que reportaron el efecto de interferencia en las personas sin alexitimia pudo deberse a un efecto "rápido", al presentar estímulos en bloques mixtos (Sánchez y Serrano, 1997; Mueller et al., 2006). 
Teniendo en cuenta las diferencias metodológicas en la tarea de Stroop emocional, además de las dificultades de interacción social asociadas con las personas con alexitimia, el presente estudio tiene como objetivo identificar el efecto de tres tipos de palabras socio-emocionales (insultos, elogios y neutras) en personas con alto y bajo puntaje de alexitimia por medio de un Stroop emocional presentado por bloques. Adicionalmente, se realizó una evaluación de las características clínicas de depresión, ansiedad y fobia social de los participantes, las cuales han sido también vinculadas con la alexitimia (Grynberg et al., 2010; Edel et al., 2010; Hamaideh, 2017).

Nosotros esperamos encontrar un efecto de interferencia en las personas con alexitimia, debido a la activación que pudieran provocar los estímulos socio-emocionales al ser presentados por bloques (efecto "lento" del Stoop). Además, suponemos que habrá un mayor nivel de los síntomas clínicos (depresión, ansiedad y fobia social), en los participantes con alta alexitimia en comparación con los de baja alexitimia, debido a la asociación reportada en estudios previos (Grynberg et al., 2010; Edel et al., 2010; Hamaideh, 2017).

\section{Participantes}

\section{Método}

Participaron 44 estudiantes de licenciatura clasificados en 2 grupos con base a la escala TAS-20 (Moral de la Rubia, 2008). Un grupo con alto puntaje de alexitimia GA (12 mujeres y 10 hombres) con una puntuación igual o superior a 61, y otro grupo con bajo puntaje de alexitimia GB (11 mujeres y 11 hombres) con una puntuación igual o menor a 28 en la escala TAS-20. La media de edad para GA fue de 19.1 $(\mathrm{DE}=1.8)$ y la del $\mathrm{GB}$ fue 20.0 ( $\mathrm{DE}=2.2$ ).

El procedimiento del estudio se apegó a los lineamientos de los principios éticos para la investigación con seres humanos (World Medical Association, 2001), y todos los participantes consintieron en participar voluntariamente. La investigación siguió las normas éticas que regula el ejercicio profesional, científico y académico de la psicología en México (Sociedad Mexicana de Psicología, 2017), y fue aprobado por el comité de ética local de la Facultad de Psicología (BUAP).

\section{Materiales}

Cuestionarios clínicos. Las características de alexitimia fueron evaluadas con la escala Toronto Alexithymic Scale (TAS-20) (Bagby, Parker y Taylor, 1994b). En el presente estudio, se utilizó la versión adaptada a la población mexicana (Moral de la Rubia, 2008), la cual ha demostrado buenos índices de confiabilidad $(\alpha=0.82)$.

Las demás características clínicas fueron evaluadas a través de cuestionarios que también han mostrado tener buenos índices de confiablidad para la población. La depresión fue valorada por el inventario de depresión de Beck (Beck Depression Inventory, BDI-II, Beck y otros, 1996) con un índice de confiabilidad de 0.87 (González et al., 2015). La ansiedad fue evaluada por la escala de ansiedad de Hamilton (Hamilton Anxiety Rating Scale HAM-A, Hamilton, 1959) con valores de confiabilidad de 0.84 (Lara et al., 1998); y la fobia social se midió con el inventario de fobia social (Social Phobia Inventory SPIN, Connor et al., 2000) que tiene un índice de confiabilidad de 0.82 (Robles-García et al., 2008).

Además, se aplicó una breve entrevista con el propósito de verificar los criterios de inclusión y exclusión. Inclusión: tener edad entre 18 a 25 años y un nivel de escolaridad mínimo de 12 años. Exclusión: personas que reportaron antecedentes de daño neurológico, enfermedades psiquiátricas, consumo de sustancias tóxicas o farmacológicas.

Estímulos. Para obtener las palabras socio-emocionales conforme al contexto, se realizó un estudio piloto del cual se seleccionaron los estímulos. El estudio consistió en solicitar a 197 estudiantes de distintas licenciaturas una lista palabras de 15 elogios, 15 insultos y 15 estímulos neutros (sustantivos). Las indicaciones fueron las siguientes: "En una hoja escribe una lista de 15 elogios o más, que al escucharlos puedan generarte sensaciones agradables". Luego de realizar la lista, se pidió a los participantes que colocarán al lado de cada palabra una calificación entre 1 y 9 , en función de la intensidad de agrado que les provocaba cada palabra, siendo 1 el puntaje menor y 9 el de mayor intensidad. Esto se realizó con la finalidad obtener el puntaje de activación subjetiva de las palabras que ellos mismos proporcionaron. Después de calificar la lista de elogios, se les pidió que realizaran el mismo procedimiento para generar una lista de 15 insultos, y que al escucharlos generaran sensaciones desagradables. Finalmente, se solicitó que realizaran una lista de 15 palabras neutras con la indicación de que fueran sustantivos (cosas u objetos), que al escucharlos no generaran sensaciones agradables ni desagradables. 
Se seleccionaron los 25 estímulos más frecuentes y con mayor activación de cada categoría para conformar los bloques de elogios, insultos y neutros. Cada palabra fue repetida 4 veces con color distinto y los estímulos de insultos y elogios fueron seleccionados por género. La puntuación media de la activación para los elogios fue de 7.4 en ambos géneros, mientras que la puntuación para los insultos fue de 6.5 para las mujeres y 6.3 para los hombres. El valor de la media de los estímulos neutros fue de 5 y se emplearon los mismos para ambos géneros. Los resultados de los análisis estadísticos corroboraron la ausencia de diferencias en el puntaje de activación subjetiva entre género para elogios [t $(24)=.027, p=.97]$ y para insultos [t $(24)=.989, p=.33$ ]

\section{Procedimiento}

El estudio se llevó a cabo en un aula académica aislada para evitar distracciones. La sesión comenzó con una breve explicación del procedimiento y una entrevista para identificar los criterios de inclusión y exclusión. Posteriormente, se aplicaron las escalas clínicas y una tarea de ensayo de la prueba de Stroop. A continuación, se aplicó la prueba del Stroop emocional y se solicitó a los participantes que respondieran lo más rápido posible conforme al color de las palabras que se mostraban en la pantalla (verde, azul, rojo y amarillo), haciendo caso omiso de la semántica. Las respuestas se realizaron presionando las teclas del ordenador que contenían los 4 colores distintos. Los estímulos se administraron en 3 bloques (elogios, Insultos y neutros) de manera contrabalanceada. La duración aproximada de cada bloque fue de 3 minutos. Se dio 1 minuto de descanso entre cada bloque. En total se mostraron 300 (100 por categoría) estímulos que fueron presentados en un sistema operativo Windows 7 , en el programa E-prime 2.0. Las palabras aparecían en un monitor de 21 pulgadas, y el tamaño de las palabras fue de 2.5 centímetros. La distancia entre el monitor y las personas fue de 70 centímetros.

\section{Análisis de datos}

Para evaluar la distribución de los datos se aplicaron las pruebas de Kolmogorov-Smirnov y Shapiro-Wilk, y para determinar la homocedasticidad de la muestra se aplicó la prueba de Levene. Las características de los participantes como la edad y el puntaje de las pruebas clínicas se compararon entre grupos mediante el estadístico $t$ de student para muestras independientes. Para analizar los tiempos de reacción de la prueba de Stroop se realizó un ANOVA de 2 factores (2x3): como factores inter-sujetos se introdujeron los grupos: alta $(>61)$ y baja alexitimia $(<24)$, y como factores intra-sujetos fueron: insultos, elogios y neutro. La corrección de Greenhouse-Geisser fue aplicada con base a los resultados de los datos, y se utilizó la prueba post-hoc Bonferroni para las comparaciones por pares.

\section{Resultados}

Los resultados de la prueba Levene arrojó un valor mayor de $p>.05$, lo cual permite aceptar la hipótesis nula por la suposición de igualdad de las varianzas. En las pruebas de normalidad Kolmogorov-Smirnov y Shapiro-Wilk, también se obtuvo un valor superior a $p>.05$, que indica que la distribución de los datos obtenidos es normal. Sin embargo, lo anterior se observó solo para el grupo de baja alexitimia, por lo que en los análisis de ANOVA se aplicó la corrección de Greenhouse-Geisser.

En los resultados de las características de los participantes, se corroboró la ausencia de diferencias entre grupos con respecto a la edad $[t(42)=-1.554, p=.12]$. Asimismo, los análisis confirmaron las diferencias significativas entre los grupos con base al TAS-20 [ $t(42)=27.972, p=<0.001]$, con valores mayores en GA que el GB.

Por su parte, en las pruebas clínicas se observaron diferencias significativas entre grupos en todas las escalas: $\mathrm{BDI}[t(42)=32.5, p=<0.001]$; HAM-A $[t=(42)=6.02, p=<0.001]$; SPIN $[t(54)=6.18$, $p=<0.001]$. Particularmente, se mostró que el GA tuvo mayores puntuaciones que el GB $(p<.01)$. Los datos se muestran de manera sintetizada en la siguiente tabla (tabla 1).

Con respecto a los resultados de la prueba Stroop emocional, el promedio del número de errores fue muy bajo $(m=1.9)$, por lo que no se encontraron diferencias entre grupos $\left[F_{(1,42)}=.038 ; p=0.84\right.$, $\eta 2=0.001]$, emociones $\left[F_{(1,42)}=.517 ; p=0.577, \eta^{2}=0.012\right]$, ni la en la interacción de grupo $X$ emociones $\left[F_{(1,42)}=2.487 ; p=0.096, \eta^{2}=0.056\right]$. 
Tabla 1

Media y desviación típica de las características de los grupos.

\begin{tabular}{lcccc}
\hline & GA & & $\mathrm{GB}$ & \\
& $\mathrm{N}=22$ & & $\mathrm{~N}=22$ & \\
\hline & Media & Desviación tipica & Media & Desviación típica \\
Edad & 19.1 & 1.8 & 20.0 & 2.1 \\
TAS-209** & 67.6 & 6.0 & 15.5 & 5.0 \\
BDI $^{* *}$ & 11.5 & 5.1 & 2.8 & 2.8 \\
HARS $^{* *}$ & 24.4 & 8.3 & 9.4 & 5.7 \\
SPIN $^{* *}$ & 33.0 & 15.3 & 11.0 & 6.5 \\
\hline Nota ${ }^{* *} p<0.01$ & & & &
\end{tabular}

Asimismo, en los resultados del tiempo de reacción tampoco se observaron diferencias entre grupos $[F(1,42)=2.504 ; p=0.12, \eta 2=0.05]$. Sin embargo, sí se observó un efecto significativo en el factor de emociones $\left[F_{(1,42)}=4.592 ; p=0.01, \eta^{2}=0.09\right]$, siendo menor el tiempo de reacción para la condición neutral que para los elogios $(p<.05)$ e insultos $(p<.05)$. Además, se presentaron diferencias significativas en la interacción de grupo X emociones $\left[F_{(1,42)}=3.683 ; p=0.02, \eta^{2}=0.08\right]$. El análisis post-hoc indicó que las palabras socio-emocionales de elogios $(p<0.01)$ e insultos $(p<0.01)$ presentaron mayor tiempo de reacción en comparación con la condición neutra en el GA, mientras que en el GB no hubo diferencias entre condiciones $(p=1.0)$. Estos resultados se muestran en la figura 1.

\section{Figura 1}

Media y desviación típica del tiempo de reacción de cada grupo ante las tres condiciones socio-emocionales. GA: Grupo con alta alexitimia; GB: Grupo con baja alexitimia. ${ }^{*} p<0.01$

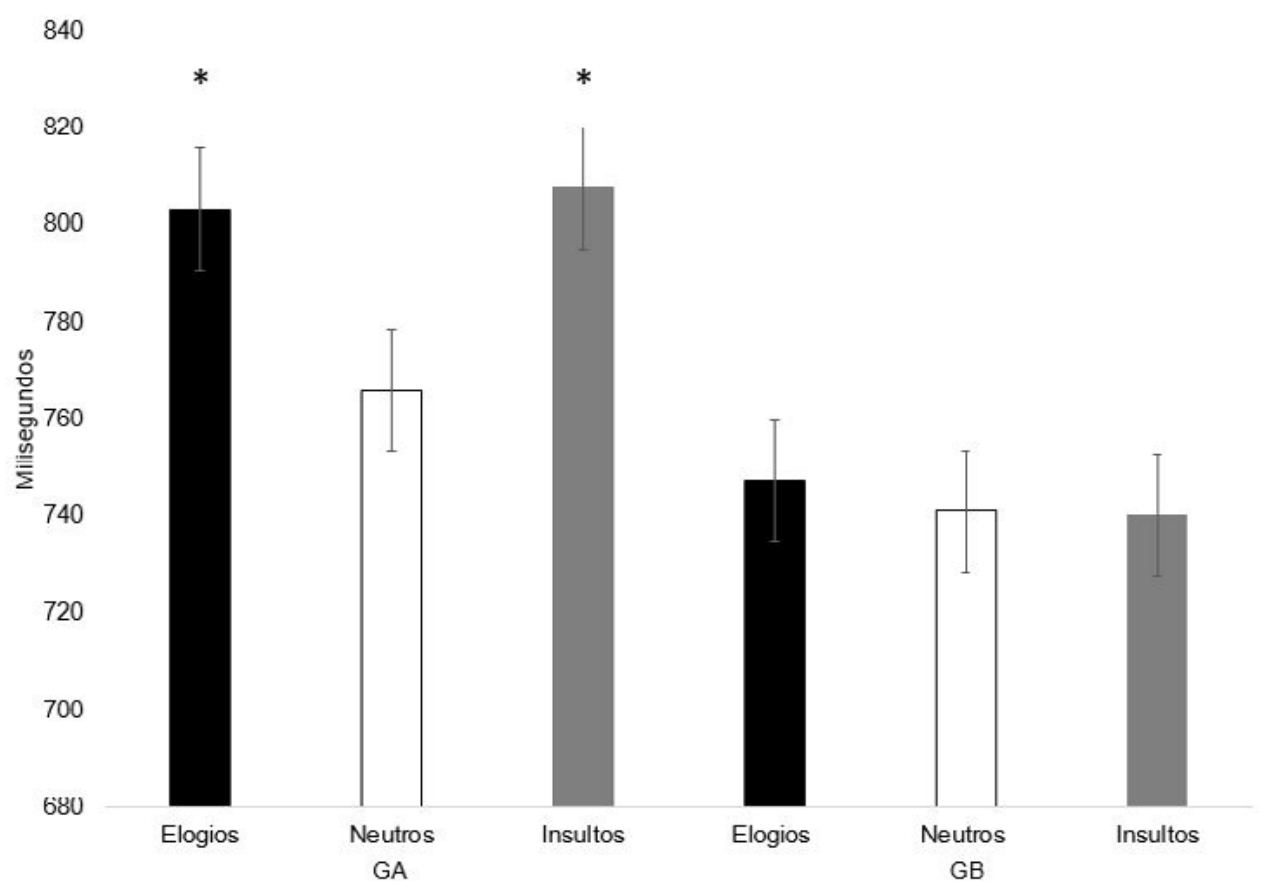

\section{Discusión}

El estudio tuvo como objetivo evaluar el efecto de las palabras socio-emocionales (insultos y elogios) en personas con alto y bajo puntaje de alexitimia conforme al TAS-20. Interesantemente, los resultados mostraron que los participantes del GA tuvieron un efecto de interferencia en el Stroop emocional ante las palabras socio-emocionales en comparación con el GB. Por lo que, los resultados sugieren que el GA presenta un sesgo atencional que no se observa en el GB. Además, los participantes del GA presentaron también un mayor puntaje en las escalas clínicas que los del GB, a pesar de no ser pacientes psiquiátricos. Así, los resultados del actual estudio muestran que ambos grupos son distintos con base a sus características clínicas y en el procesamiento perceptual ante estímulos lingüísticos socio-emocionales (insultos y elogios) evaluados a través del Stroop emocional. 
Características de los participantes

En el presente estudio las variables de la edad y la escolaridad fueron pareadas por grupos. Además, se excluyeron a los participantes que reportaron antecedentes de daño neurológico, enfermedades psiquiátricas, consumo de sustancias tóxicas o farmacológicas. De este modo, los resultados que fueron obtenidos no se atribuyen a dichas variables.

Con respecto a las características clínicas, encontramos que hubo diferencias entre grupos. Lo anterior se observó incluso cuando en el estudio no se consideraron a personas con antecedentes psiquiátricos. Así, los resultados encontrados se muestran a favor de trabajos previos que han vinculado la alexitimia con síntomas clínicos (Leweke et al., 2012; Taylor, Bagby, y Parker, 1997). Específicamente, con la depresión (Leweke et al., 2012; Saarijärvi et al., 2006; Tolmunen et al., 2010), la ansiedad (Hamaideh, 2017), y la fobia social (Edel et al., 2010).

\section{Stroop emocional (errores y tiempo de reacción)}

En relación con los errores, no se observaron diferencias significativas, lo cual ha sido reportado en estudios previos (Mueller et al., 2006). Con respecto a los tiempos de reacción se encontró un efecto de interferencia en el GA, con un mayor tiempo de reacción que el GB ante las palabras de elogios e insultos. Lo anterior es consistente con otros estudios que han reportado resultados similares ante estímulos activadores (Pandey Rakesh, 1995; Parker et al., 1993), pero no se había demostrado ante palabras socio-emocionales. De este modo, los resultados encontrados en este estudio permiten extender que el efecto de interferencia observado en personas con alto puntaje de alexitimia puede presentarse también ante estímulos socio-emocionales de elogios e insultos.

De acuerdo con otros autores, la explicación del efecto de interferencia del Stroop emocional puede deberse a la exposición de estímulos emocionales que son interpretados como amenazantes (Algom et al., 2004). Los estímulos amenazantes pueden activar un mecanismo cerebral que actúa de manera automática conocido como vigilancia automática, o captura automática de la atención. Según este modelo, el mecanismo prioriza los recursos hacia los estímulos amenazantes, lo que precipita la interrupción temporal de cualquier otra actividad en curso (Algom et al., 2004; Mueller et al., 2006). En otras palabras, es un sistema cerebral básico que inicia en el sistema subcortical límbico-emocional hacia la corteza (botton-up). Particularmente, en el caso del Stroop emocional, la actividad interrumpida por los estímulos amenazantes correspondería a la actividad de nombrar los colores. Así, es posible interpretar que las palabras socio-emocionales de elogios e insultos fueron activadoras o amenazantes para el GA, lo que propició un retraso en sus respuestas.

En relación con lo anterior, algunos autores han propuesto que las personas con alexitimia tienen una alta sensibilidad de activación automática y una posible deficiencia para modular respuestas emocionales (Pandey Rakesh, 1995; Parker et al., 1993; Lundh y Simonsson-Sarnecki, 2002). Interesantemente, en el estudio presente el efecto de interferencia no se presentó en el GB, lo cual coincide que el estímulo socio-emocional tuvo un mayor efecto en las personas con alta que con baja alexitimia. En esta línea, un estudio reciente reportó que los estimulos sociales pueden influir de distinta forma la respuesta fisiológica de las personas con y sin alexitimia (Martínez-Velázquez et al., 2017). Desafortunadamente, en el presente estudio no se tomaron mediciones fisiologicas, por lo que es necesario corroborar el efecto activador de las palabras socioemocionales en estudios posteriores.

Otra posible explicación podría ser que los participantes con alta alexitimia presentan un sesgo atencional que está vinculado con las palabras que para ellos pueden ser más practicadas, familiares (Bremner et al., 2004; Thomson, Patel, Platek, y Shackelford, 2015), o incluso llamativas (loannou et al., 2016). Aunque las dificultades socio-emocionales que han sido asociadas con las personas con alexitimia (Mallinckrodt y Wei, 2005; Rozenstein, Stein, y Latzer, 2018) no favorecen esta interpretación, cabe la posibilidad de que las personas con alta alexitimia presten mayor atención que las de baja alexitimia hacía los estímulos socio-emocionales por ser más llamativos que los neutros, independientemente de su valencia.

Por otro lado, el contraste de los resultados entre el presente estudio y otros (Sánchez y Serrano, 1997; Mueller et al., 2006) podría ser explicado por las diferencias metodológicas. En primer lugar, el uso de los estímulos socio-emocionales que son originales de este estudio. Segundo, la presentación de los estímulos que aquí presentamos fue en bloques por categoría, y no en bloques mixtos. Estudios previos han demostrado que la desaceleración en el nombramiento de palabras emocionales se elimina cuando tales palabras se mezclan en categorías de sucesión aleatoria (Algom et al., 2004). Así, los hallazgos obtenidos en el presente estudio se mostraron a favor de que el efecto "lento" del Stroop emocional se presenta principalmente en las personas con alta alexitimia. Mientras que el efecto "rápido" parece afectar esencialmente a las personas con baja alexitimia (Sánchez y Serrano, 1997; Mueller et al., 2006). No obstante, es necesario corroborar estas especulaciones con más estudios futuros. 


\section{Conclusiones}

Hasta donde tenemos conocimiento, el presente estudio es el primero en evaluar la percepción de personas con alto y bajo nivel de alexitimia utilizando palabras socio-emocionales (insultos y elogios) en una prueba de Stroop emocional. Los hallazgos mostraron un efecto de interferencia en las personas con alta alexitimia en comparación con baja alexitimia, lo que sugiere un sesgo atencional hacia los estímulos socio-emocionales de insultos y elogios. Lo anterior podría estar vinculado con las dificultades que muestran las personas con alexitimia para adaptarse socialmente. También encontramos que las personas que presentaron mayor nivel de alexitimia presentaron mayor nivel de depresión, ansiedad y fobia social. El valor de los resultados no puede ser atribuido a las diferencias de edad, escolaridad, el consumo de fármacos o antecedentes de enfermedades psiquiátricas.

Algunas de las limitaciones del presente estudio fueron el tamaño de la muestra, la cual puede ser mas grande en estudios posteriores. Asimismo, sería conveniente que en estudios futuros se puedan implementar técnicas de medición de la activación fisiológica durante la evaluación o posterior a la misma y así poder vincularla con el sesgo atencional que se observó en el presente estudio.

\section{Referencias}

1. Bagby, R. M., Parker, J. D., y Taylor, G. J. (1994). The twenty-item Toronto alexithymia scale-I. Item selection and cross-validation of the factor structure. Journal of Psychosomatic Research, 38, 23-32. https://doi.org/10.1016/0022-3999(94)90005-1

2. Bermond, B., Oosterveld, P., y Vorst, H. C. M. (2015). Measures of Alexithymia. In G. J. Boyle, D. H. Saklofske, y G. Matthews (Eds.), Measures of personality and social psychological constructs, (pp. 227-256). San Diego: Academic.

3. Bermond, B., Vorst, H. C. M., y Moormann, P. P. (2006). Cognitive neuropsychology of alexithymia: Implications for personality typology. Cognitive Neuropsychiatry, 11, 332-360. https://doi. org/10.1080/13546800500368607

4. Bremner, J. D., Vermetten, E., Vythilingam, M., Afzal, N., Schmahl, C., Elzinga, B., y Charney, D. S. (2004). Neural correlates of the classic color and emotional Stroop in women with abuse-related posttraumatic stress disorder. Biological Psychiatry, 55, 612-620. https://doi.org/10.1016/J. BIOPSYCH.2003.10.001

5. Calderon, S., Samstag, L. W., Papouchis, N., y Saunders, B. A. (2019). The effects of early parental death and grief on interpersonal functioning and alexithymia in adults. Psychopathology, 52, 1-7. https://doi.org/10.1159/000501156

6. Carretié, L., Hinojosa, J. A., Albert, J., López-Martín, S., De La Gándara, B. S., Igoa, J. M., y Sotillo, M. (2008). Modulation of ongoing cognitive processes by emotionally intense words. Psychophysiology, 45, 188-196. https://doi.org/10.1111/j.1469-8986.2007.00617.x

7. Connor, K. M., Davidson, J. R. T., Churchill, L. E., Sherwood, A., Foa, E. y Weisler, R. H., (2000). Psychometric properties of the Social Phobia Inventory (SPIN) - New self-rating scale. British Journal of Psychiatry, 176, 379-386. https://doi.org/10.1192/bjp.176.4.379

8. Cook, R., Brewer, R., Shah, P., y Bird, G. (2013). Alexithymia, not autism, predicts poor recognition of emotional facial expressions. Psychological Science, 24, 723-732. https://doi. org/10.1177/0956797612463582

9. Donges, U.-S., Kersting, A., y Suslow, T. (2014). Alexithymia and perception of emotional information: A review of experimental psychological findings. Universitas Psychologica, 13, 745-756. https://doi.org/10.11144/Javeriana.UPSY13-2.apei

10. Edel, M. A., Rudel, A., Hubert, C., Scheele, D., Brüne, M., Juckel, G., y Assion, H. J. (2010). Alexithymia, emotion processing and social anxiety in adults with ADHD. European Journal of Medical Research, 15, 403. https://doi.org/10.1186/2047-783x-15-9-403

11. Freyberger, H., Kunsebeck, H. W., Lempa, W., Wellmann, W., y Avenarius, H. J. (1985). Psychotherapeutic Interventions in alexithymic patients. With special regard to ulcerative colitis and Crohn patients. Psychotherapy and Psychosomatics, 44, 72-81. https://doi.org/10.1159/000287896

12. Goerlich-Dobre, K. S., Bruce, L., Martens, S., Aleman, A., y Hooker, C. I. (2014). Distinct associations of insula and cingulate volume with the cognitive and affective dimensions of alexithymia. Neuropsychologia, 53, 284 -292. doi.org/10.1016/j.neuropsychologia.2013.12.006

13. González, D., Reséndiz, A., y Reyes-Lagunes, I. (2015). Adaptation of BDI-II in Mexico. Salud Mental, 38, 237-244. https://doi.org/10.17711/SM.0185-3325.2015.033

14. Grynberg, D., Chang, B., Corneille, O., Maurage, P., Vermeulen, N., Berthoz, S., y Luminet, O. (2012). Alexithymia and the processing of emotional facial expressions (EFEs): Systematic review, unanswered questions and further perspectives. PLOS ONE, 7, e42429. https://doi.org/10.1371/ journal.pone.0042429 
15. Grynberg, D., Luminet, O., Corneille, O., Grezes, J., y Berthoz, S. (2010). Alexithymia in the interpersonal domain: A general deficit of empathy? Personality and Individual Differences, 49, 845-850. https://doi.org/10.1016/j.paid.2010.07.013

16. Hamaideh, S. H. (2018). Alexithymia among Jordanian university students: Its prevalence and correlates with depression, anxiety, stress, and demographics. Perspectives in Psychiatric Care, 54, 274-280. https://doi.org/10.1111/ppc.12234

17. Haviland, M.G., Warren, W.L., Riggs, M.L. (2000). An observer scale to measure alexithymia. Psychosomatics; 41, 385-92. https://doi.org/10.1176/appi.psy.41.5.385

18. Hobson, H., Hogeveen, J., Brewer, R., Catmur, C., Gordon, B., Krueger, F. y Grafman, J. (2018). Language and alexithymia: Evidence for the role of the inferior frontal gyrus in acquired alexithymia. Neuropsychologia, 111, 229-240. https://doi.org/10.1016/J.NEUROPSYCHOLOGIA.2017.12.037

19. Ioannou, S., Morris, P.,Terry, S., Baker, M., Gallese, V., y Reddy V. (2016). Sympathy crying: insights from infrared thermal imaging on a female sample. PLOS ONE 11, e0162749. https://doi. org/10.1371/journal.pone.0162749

20. Kissler, J., Assadollahi, R., y Herbert, C. (2006). Emotional and semantic networks in visual word processing: Insights from ERP studies. Progress in Brain Research, 156, 147-183. https://doi. org/10.1016/S0079-6123(06)56008-X

21. Lara, M.C., Ontiveros, M., Berlanga, C., Fuente, J.R., (1988). Diferencias entre crisis de angustia y ansiedad generalizada en la escala de Hamilton para ansiedad. Salud Mental, 11, 7-10. Recuperado de: http://repositorio.inprf.gob.mx/bitstream/handle/123456789/5532/sm110407. pdf? sequence $=1$ \&isAllowed $=y$

22. Luminet, O., Rokbani, L., Ogez, D., y Jadoulle, V. (2007). An evaluation of the absolute and relative stability of alexithymia in women with breast cancer. Journal of Psychosomatic Research, 62, 641-648. https://doi.org/10.1016/j.jpsychores.2007.01.003

23. Lundh, L. G., y Simonsson-Sarnecki, M. (2002). Alexithymia and cognitive bias for emotional information. Personality and Individual Differences, 32, 1063-1075. https://doi.org/10.1016/ S0191-8869(01)00110-6

24. Mallinckrodt, B., y Wei, M. (2005). Attachment, social competencies, interpersonal problems, and psychological distress. Journal of Counseling Psychology, 52(3), 358-367. https://doi. org/10.1037/0022-0167.52.3.358

25. Martínez-Velázquez, E. S., Honoré, J., De Zorzi, L., Ramos-Loyo, J., y Sequeira, H. (2017). Autonomic reactivity to arousing stimuli with social and non-social relevance in alexithymia. Frontiers in psychology, 8, 361. https://doi.org/10.3389/fpsyg.2017.00361

26. McKenna, F. P., y Sharma, D. (2004). Reversing the emotional Stroop effect reveals that it is not what it seems: The role of fast and slow components. Journal of Experimental Psychology: Learning, Memory, and Cognition, 30, 382-392. https://doi.org/10.1037/0278-7393.30.2.382

27. Moral de la Rubua, J. (2008). Propiedades psicométricas de la escala de alexitimia de Toronto de 20 reactivos en México. Revista Electronica de Psicología Iztacala, 11, 97-114. Recuperado de https://www.iztacala.unam.mx/carreras/psicologia/psiclin/vol11num2/art5-vol11-No2.pdf

28. Mueller, J., Alpers, G. W., y Reim, N. (2006). Dissociation of rated emotional valence and Stroop interference in observer-rated alexithymia. Journal of Psychosomatic Research, 61, 261-269. https://doi.org/10.1016/J.JPSYCHORES.2006.02.017

29. Pandey R. (1995). Stroop interference effect of emotion-arousing words in alexithymia. Journal of the Indian Academy of Applied Psychology, 21, 21-28. Recuperado de: https://psycnet.apa.org/ record/1996-14488-001

30. Parker, J. D. A., Taylor, G. J., y Bagby, R. M. (1993). Alexithymia and the processing of emotional stimuli: An experimental study. New Trends in Experimental and Clinical Psychiatry, 9, 9-14. Recuperado de: https://psycnet.apa.org/record/1994-13552-001

31. Picardi, A., Fagnani, C., Gigantesco, A., Toccaceli, V., Lega, I., y Stazi, M. A. (2011). Genetic influences on alexithymia and their relationship with depressive symptoms. Journal of Psychosomatic Research, 71, 256-263. https://doi.org/10.1016/J.JPSYCHORES.2011.02.016

32. Robles-García R., Espinosa-Flores, R.A., Padilla-Guitiérrez, A.C., Álvarez-Rojas, M.A., y Páez-Agraz, F. (2008). Ansiedad social en estudiantes universitarios: Prevalencia y variables psicosociales relacionadas. Psicología Iberoamericana, 16, 54-63. Recuperado de: https://www. redalyc.org/articulo.oa?id $=133920328008$

33. Rozenstein, M. H., Stein, D., y Latzer, Y. (2018). Subjective and Objective: Alexithymia and Social Cognition in Eating Disorders. The Israel journal of psychiatry and related sciences, 55, 45-54. Recuperado de: https://cdn.doctorsonly.co.il/2018/06/07 Subjective-and-Objective.pdf 
34. Saarijärvi, S., Salminen, J. K., y Toikka, T. (2006). Temporal Stability of Alexithymia Over a FiveYear Period in Outpatients with Major Depression. Psychotherapy and Psychosomatics, 75, 107-112. https://doi.org/10.1159/000090895

35. Martínez-Sánchez, F., y Marín, J. (1997). Influencia del nivel de alexitimia en el procesamiento de estímulos emocionales en una tarea Stroop. Psicothema, 9, 519-527. Recuperado de: https:// www.redalyc.org/pdf/727/72709306.pdf

36. Sifneos, P. E. (1973). The prevalence of "alexithymic" characteristics in psychosomatic patients. Psychotherapy and Psychosomatics, 22(2-6), 255-262. https://doi.org/10.1159/000286529

37. Taake, I., Jaspers-Fayer, F., y Liotti, M. (2009). Early frontal responses elicited by physical threat words in an emotional Stroop task: Modulation by anxiety sensitivity. Biological Psychology, 81(1), 48-57. https://doi.org/10.1016/J.BIOPSYCHO.2009.01.006

38. Taylor, G. J., y Bagby, R. M. (2013). Psychoanalysis and Empirical Research: The example of alexithymia. Journal of the American Psychoanalytic Association, 61, 99-133. https://doi. org/10.1177/0003065112474066

39. Taylor, G. J., Bagby, R. M., y Parker, J. D. A. (1997). Disorders of affect regulation : alexithymia in medical and psychiatric illness. Cambridge, UK: Cambridge University Press.

40. Thomson, J. W., Patel, S., Platek, S. M., y Shackelford, T. K. (2015). Sex differences in implicit association and attentional demands for information about infidelity. Evolutionary Psychology, 5, 569-583. https://doi.org/10.1177/147470490700500307

41. van der Velde, J., van Tol, M.J., Goerlich-Dobre, K. S., Gromann, P. M., Swart, M., de Haan, L., ... y Aleman, A. (2014). Dissociable morphometric profiles of the affective and cognitive dimensions of alexithymia. Cortex, 54, 190-199. https://doi.org/10.1016/J.CORTEX.2014.02.017

42. Vanheule, S., Desmet, M., Rosseel, Y., y Verhaeghe, P. (2007). Relationship patterns in alexithymia: A Study using the Core Confl ictual Relationship Theme (CCTR) method. Psychopathology, 40, 14-21. https://doi.org/10.1159/000096385

43. Vorst, H. C. M., y Bermond, B. (2001). Validity and reliability of the Bermond-Vorst Alexithymia Questionnaire. Personality and Individual Differences, 30, 413-434. https://doi.org/10.1016/ S0191-8869(00)00033-7

44. World Medical Association. (2001). World Medical Association Declaration of Helsinki. Ethical principles for medical research involving human subjects. Bulletin of the World Health Organization, 79, 373. Recuperado de: https//www.wma.net/en/30publications/10policies/b3/index.html (accessed July 8, 2010)

Agradecimientos: Agradecemos de manera muy especial a las estudiantes Pamela Ponce y Valeria Pérez por su valioso apoyo para el reclutamiento de los participantes.

RECIBIDO: 14 de septiembre de 2019

MODIFICADO: 5 de noviembre de 2019

ACEPTADO: 18 de julio de 2020 